\title{
Action Spectroscopy of Isomer-Selected Luciferin Anions
}

\author{
Christina Kjær - James N. Bull · Eduardo Carrascosa • Steen Brøndsted \\ Nielsen • Evan J. Bieske
}

Received: date / Accepted: date

\begin{abstract}
Luciferin molecules are common luminophores found throughout the biological kingdoms. Here, electrospray ionization and tandem ion mobility spectrometry coupled with laser spectroscopy are used to demonstrate that D-luciferin and oxyluciferin deprotonated anions can be produced in two isomeric forms, which can be separated by virtue of their different collision cross-sections with a buffer gas. The two isomers possess distinguishable but partially overlapping photodepletion action spectra over the visible range, implying distinct intrinsic absorption profiles. The site of deprotonation and tautomeric forms of the electrosprayed isomers are assigned through comparisons between experimental and calculated collision cross-sections and electronic excitation energies. The study clearly shows that electrospray ionization of biochromophore molecules can generate multiple isomeric forms with distinct electronic spectra.
\end{abstract}

Keywords Ion mobility · Deprotomers · Tautomers · Photodepletion spectroscopy

C. Kjær \& S. Brøndsted Nielsen

Department of Physics and Astronomy, Aarhus University, Aarhus 8000, Denmark

\section{J. N. Bull}

School of Chemistry, Norwich Research Park, University of East Anglia, Norwich NR4 7TJ, United Kingdom

E. Carrascosa

Bruker Daltonik GmbH, Fahrenheitstrasse 4, 28359 Bremen, Germany

E. J. Bieske

School of Chemistry, University of Melbourne, Parkville, VIC 3010, Australia

\section{Introduction}

Bioluminescence is the generation of light by enzymecatalyzed chemiluminescent reactions in living organisms. It is a common phenomenon throughout the biological kingdoms, $[1,2]$ including in marine organisms such as jellyfish, squid and deep sea fish, terrestrial organisms such as luminescent fungi, the Photorhabdus luminescens bacterium and arthropods including fireflies, glow worms and click beetles. Over the last decade, there has been growing interest in developing luciferinbased luminophores and luciferase enzymes as probes for in vitro and in vivo imaging of tissue and cellular processes, including assaying tumour cells in animals, and for monitoring gene regulation and biomolecule binding dynamics.[3, 4, 5] The ongoing need for a diverse toolkit of fluorescent biomarkers requires a detailed understanding of the fundamental photophysics of luminophore molecules and how a solvent or protein environment modifies these photophysics. $[6,7,8]$

In fireflies, bioluminescence involves a four step mechanism in which a luciferin molecule is catalytically oxidized with adenosine triphosphate (ATP) and $\mathrm{O}_{2}$ to form oxyluciferin in its first singlet excited state $\left(\mathrm{S}_{1}\right)$. This then decays through radiative emission generating bioluminescence. In turn, enzymes convert oxyluciferin back to luciferin so that the cycle may repeat.[9] Intriguingly, all species of firefly have a common luciferin luminophore, although each species has a distinct bioluminescence spectrum due to species-dependent host enzymes and concentrations of other biomolecules in the vicinity of the luciferase enzyme. $[1,10,11,12,13$, 14] For example, the bioluminescence spectrum from the Pennsylvania firefly Photuris pennsylvanica peaks at $538 \mathrm{~nm}$ (aqua-green light), while the spectrum for the eastern firefly Photinus pyralis peaks at $562 \mathrm{~nm}$ 
(yellow-green light), and the spectrum for the Japanese firefly Luciola cruciata peaks at $550 \mathrm{~nm}$ (green light). The bioluminescence spectrum from the railway worm Phrixotrix hirtus, which has the same luminophore, peaks at $623 \mathrm{~nm}$.[15] Laboratory studies have shown that the bioluminescence spectra from firefly luciferins are $\mathrm{pH}$-sensitive (red-shifted at low $\mathrm{pH}$ ), $[15,16]$ whereas the bioluminescence spectra of beetle luciferases are insensitive to $\mathrm{pH}$. The origin for the $\mathrm{pH}$ sensitivity of firefly bioluminescence spectra is unclear, although a current hypothesis is that there are species-dependent enzyme binding pockets and specific protomeric and tautomeric forms of the luciferin luminophore that are favored over certain $\mathrm{pH}$ ranges $[15,17]$.

One of the ultimate goals in the investigation of biochromophores and photoactive proteins is to understand how the structure of a host enzyme/protein modifies the chromophore's intrinsic photophysics. This understanding may be developed in a 'bottom-up' approach, starting from benchmark measurements on the transition energies and photochemical dynamics of gas-phase luciferin molecules. Gas-phase measurements are also readily compared with quantum chemical calculations, which are usually straightforward to carry out on isolated molecules.

In a step towards probing the intrinsic photophysics of luciferin molecules (Fig. 1), Brøndsted Nielsen and co-workers[18, 19] used photodissociation action spectroscopy to infer the electronic absorption spectra for a series of deprotonated luciferin anions. The first of these studies reported photodissociation spectra spanning the $450-580 \mathrm{~nm}$ range for deprotonated Dluciferin $\left(\mathrm{LH}^{-}\right)$, which were recorded using two separate instruments at Aarhus University (Sep1 and ELISA). The photodissociation spectra were assigned to the carboxylate deprotomer based on: (i) calculations at the B3LYP/TZVPP level of theory suggesting it is $21 \mathrm{~kJ} \mathrm{~mol}^{-1}$ lower in energy than the phenoxide deprotomer, and (ii) because TD-DFT calculations of the excitation wavelengths at the B3LYP/TZVPP ++ level of theory for the carboxylate deprotomer agreed better with the maximum response in the photodissociation action spectra.[18] Interestingly, the peak wavelength of the photodissociation action spectrum differed in the two experiments; measurements using the Sep1 instrument recorded anion fragments over a short acquisition window (0-3 $\mu$ s after photoexcitation) and gave maximum response at $\approx 500 \mathrm{~nm}$, whereas measurements using the ELISA instrument recorded neutral fragments formed by dissociation or electron detachment over a longer acquisition window $(33 \mu \mathrm{s}$ to $3 \mathrm{~ms}$ after photoexcitation) and gave maximum response at $\approx 540 \mathrm{~nm}$. The difference between the photodissociation spectra<smiles>O=C(O)C1CSC(c2nc3ccc(O)cc3s2)=N1</smiles><smiles>O=C1CSC(c2nc3ccc(O)cc3s2)=N1</smiles>

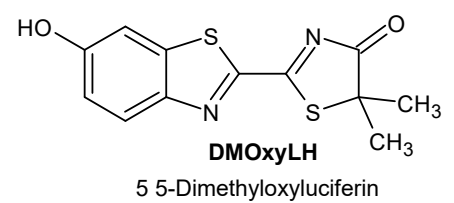

Fig. 1 Structures and naming for luciferin molecules considered in this study. Molecules are shown as their $E$ isomers with respect to the central bond connecting the two rings.

was attributed to the extent of statistical dissociation that ensued within the respective detection windows. A longer acquisition window allows detection of ions from slower statistical dissociation processes that are likely to occur following absorption of longer wavelength photons.

A subsequent study recorded photodissociation action spectra with visible light for deprotonated oxyluciferin $\left(\mathrm{OxyLH}^{-}\right)$and deprotonated 5,5dimethyloxyluciferin $\left(\mathrm{DMOxyL}^{-}\right)$as well as $\mathrm{OxyLH}^{-} \cdot \mathrm{H}_{2} \mathrm{O}$ complexes using both Sep1 and ELISA.[19] The purpose of using the 5,5dimethyloxyluciferin sample was to ensure that deprotonation was on the phenolate group by locking the five-membered ring in the keto tautomer. Both $\mathrm{OxyLH}^{-}$and $\mathrm{DMOxyL}^{-}$exhibited a broad spectral band over the $450-650 \mathrm{~nm}$ range with maximum response at $550 \mathrm{~nm}$. The maximum response for $\mathrm{OxyLH}^{-} \cdot \mathrm{H}_{2} \mathrm{O}$ complexes was blue shifted by $\approx 50 \mathrm{~nm}$.

In another study, Woodhouse et al.[20] investigated $\mathrm{LH}^{-}$and the deprotonated anion of a synthetic derivative infraluciferin using photoelectron spectroscopy at several wavelengths in the near-UV. The photoelectron spectra for $\mathrm{LH}^{-}$were assigned to the phenolate deprotomer based on B3LYP/6-311++G(3df,3pd) calculations that suggested the carboxylate deprotomer is $45 \mathrm{~kJ} \mathrm{~mol}^{-1}$ less stable. However, single-color photoelectron spectroscopy does not probe the biologically relevant $S_{1}$ state because this state is situated below the detachment threshold. More recently, Patel et al.[21] 
used a similar photoelectron spectroscopy strategy to study $\mathrm{OxyLH}^{-}$and several methylated derivatives designed to have a single deprotomer. Comparison of the photoelectron spectra for $\mathrm{OxyLH}^{-}$and the methylated forms provided strong evidence for the existence of at least two gas-phase forms, although determining the relative abundances is difficult because the photodetachment cross-sections for each deprotomer are not known. Ultimately, these measurements highlight the difficulty of performing gas-phase action spectroscopy on $\mathrm{OxyLH}^{-}$(or other luciferin molecules with multiple gas-phase isomers) without isomer-selective techniques.

The present study applies an emerging action spectroscopy technique based on coupling electrospray ionization with tandem ion mobility spectrometry (IMS) and laser spectroscopy to record isomer-selected action spectra for $\mathrm{LH}^{-}, \mathrm{OxyLH}^{-}$and $\mathrm{DMOxyL}^{-}$(Fig. 1). In IMS, charged isomers (e.g. deprotomers and tautomers) drifting under the influence of an electric field through a buffer gas are separated according to their respective drift speeds, which depend on their collision cross-section. Typically, the target isomer is selected in a primary IMS stage and then exposed to wavelength tunable light, with separation of photoisomers or photofragments in a second IMS stage. This isomer selectivity avoids complications associated with overlapping spectra of coexisting isomers. The present study demonstrates that gas-phase $\mathrm{LH}^{-}$and $\mathrm{OxyLH}^{-}$ can exist in several isomeric forms with distinct action spectra and absorption profiles over the $\mathrm{S}_{1} \leftarrow \mathrm{S}_{0}$ bands. There was no evidence for photoisomerization or phototautomerization for any of the electrosprayed isomers. This work clearly exemplifies the utility of isomer-specific techniques when studying the photochemistry of ionic biomolecules that possess several (de)protonation sites.

\section{Experimental methods}

Experiments were performed using a tandem ion mobility spectrometer coupled with an electrospray ionization source and quadrupole mass filter.[22, 23] Luciferin anions were produced through electrospray ionization of $\mathrm{a} \approx 10 \mu \mathrm{mol} \mathrm{L}{ }^{-1}$ solution of either $\mathrm{LH}_{2}$ (Sigma-Aldrich, $>99 \%$ purity), $\mathrm{OxyLH}_{2}$, or $\mathrm{DMOxyLH}$ dissolved in

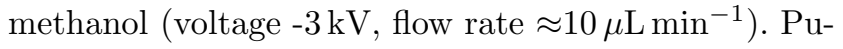
rities of the $\mathrm{OxyLH}_{2}$ and DMOxyLH samples, which were synthesized as part of an earlier study,[19] were confirmed with high-resolution mass spectrometry. In the ion mobility experiments, electrosprayed ions were transferred via a heated capillary into a radio-frequency (RF) ion funnel (source ion funnel), which radially gathered and confined the ions. An ion gate at the end of the source ion funnel injected $\approx 100 \mu$ s packets of ions at $40 \mathrm{~Hz}$ into the drift region where they were propelled by an electric field $\left(44 \mathrm{~V} \mathrm{~cm}^{-1}\right)$ through either $\mathrm{N}_{2}$ buffer gas, $\mathrm{N}_{2}$ seeded with $\approx 1 \%$ propan-2-ol, or $\mathrm{N}_{2}$ seeded with $\approx 1 \% \mathrm{SF}_{6}$ (total pressure of $\approx 6$ Torr).[24, 25, 26] Isomers were separated spatially and temporally due to differences in their collision cross-sections with the buffer gas. [27] After traversing the drift region, a second ion funnel collected the ions and introduced them into a differentially pumped octupole ion guide and quadrupole mass filter that mass selected the ions before they reached a Channeltron ion detector. The detector was connected to a multichannel scaler that produced a histogram of ion counts against arrival time, $t$, corresponding to an arrival time distribution (ATD). In all presented ATDs, $t=0$ corresponds to the opening of the first ion gate. The mobility resolution, $t / \Delta t$, for singly-charged anions is typically 80-90see Adamson et al.[22, 23] for further discussion on instrument specifications and performance.

For the action spectroscopy measurements, packets of ions with similar collision cross-sections were selected using a Bradbury-Nielsen ion gate $(\approx 100 \mu \mathrm{s}$ opening time) situated midway along the drift region. Immediately after gating, the mobility-selected ions were excited with a transverse pulse of light from an optical parametric oscillator (OPO, EKSPLA NT342B, $2 \mathrm{~mJ} \mathrm{~cm}^{-2}$ pulse $^{-1} \pm 20 \%$ over the action spectrum wavelength range). The OPO was operated at $20 \mathrm{~Hz}$, half the rate of ion injection, allowing accumulation of light-on and light-off ATDs. The difference between the light-on and light-off ATDs reflected the photoresponse (photoaction ATD). Action spectra were derived by integrating the photoaction ATD signal and normalizing with respect to light pulse fluence and total laser-off signal at each wavelength. For the major ATD peak $\mathrm{LH}^{-}$, photodetachment action spectra were recorded using $\mathrm{N}_{2}$ seeded with $\approx 1 \% \mathrm{SF}_{6}$ and monitoring the formation of $\mathrm{SF}_{6}{ }^{-}$to infer electron detachment.[25, 26] There was insufficient laser power for wavelengths longer than $\approx 650 \mathrm{~nm}$ to record action spectra.

Action spectra were recorded with light fluences that produced no more than a few percent photodepletion $\left(\approx 2 \mathrm{~mJ} \mathrm{~cm}^{-2}\right.$ pulse $\left.^{-1}\right)$ - see photoaction ATDs in the Electronic Supplementary Material. Under these conditions, photodepletion was observed although there was no appreciable photofragment signal when the quadrupole mass filter was set to act as an ion guide (i.e., no mass filtering). Note that the instrument does not transmit ions with $m / z<80$ and has reduced transmission efficiency for ions with $m / z$ 80-120. 

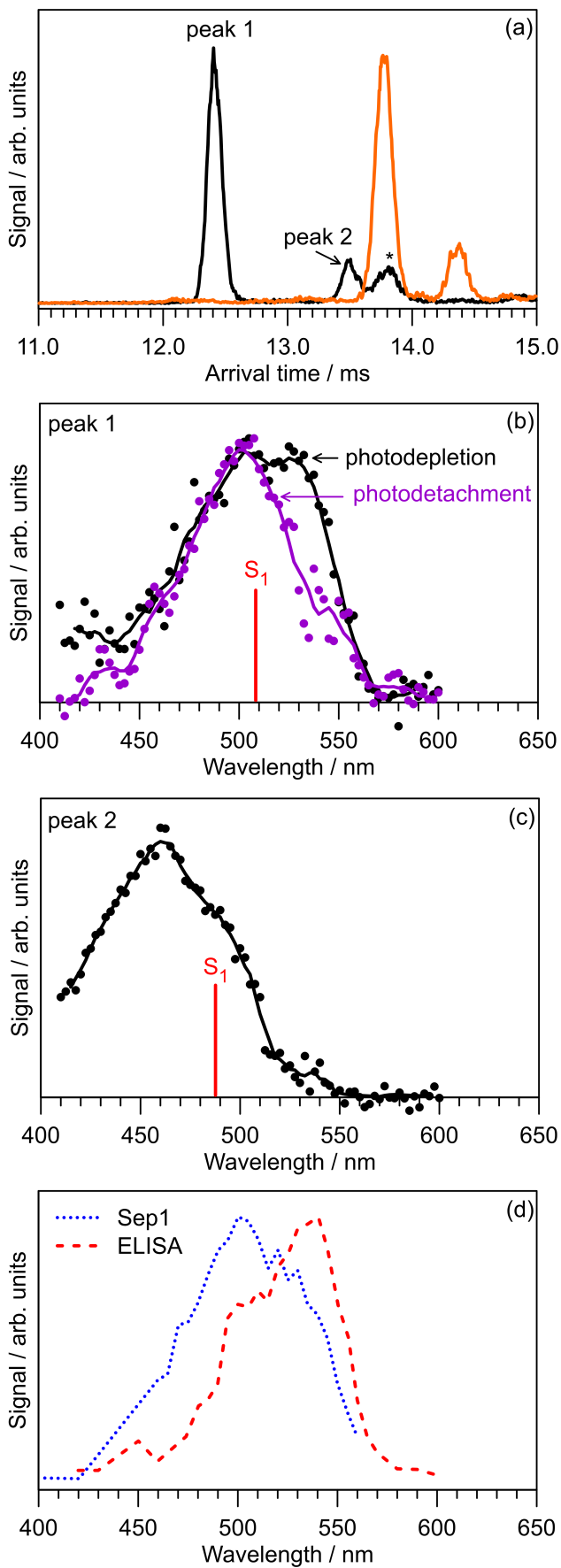

Fig. 2 (a) Arrival time distributions for $\mathrm{LH}^{-}$using $\mathrm{N}_{2}$ (black) and $\mathrm{N}_{2}+\approx 1 \%$ propan-2-ol (orange) buffer gas. The feature denoted by $*$ for the black trace originates from $\mathrm{LH}^{-} \cdot \mathrm{CH}_{3} \mathrm{OH}$ clusters that dissociate in the ion funnel at the end of the drift region (the corresponding feature in the orange trace has arrival time $>15 \mathrm{~ms}$ ). (b) Photodepletion and photodetachment action spectra for ATD peak 1 (assigned to the $E$-phenolate isomer). (c) Photodepletion action spectrum for ATD peak 2 (assigned to the $E$-carboxylateenol isomer). (d) Photodissociation spectra (smoothed) for $\mathrm{LH}^{-}$from Ref. 18. Red bars in (b) and (c) indicate STEOMDLPNO-CCSD/aug-cc-pVDZ vertical excitation wavelengths from Table 1.

\section{Computational methods}

Electronic structure calculations were carried out using the Gaussian 16 A.03,[28] ORCA 4.2.1,[29] and MRCC (Febuary 2018 release)[30] software packages. Optimized geometries, vibrational frequencies and isomerization transition states on the ground electronic state were computed at the $\omega$ B97X-D/aug-cc-pVDZ level of theory followed by single-point energy calculations at the DLPNO-CCSD(T)/aug-cc-pVDZ level of theory. [31, 32, 33] Vertical excited state energies were computed at the df-CC2/aug-cc-pVTZ and STEOMDLPNO-CCSD/aug-cc-pVDZ levels of theory. [34, 35]

Collision cross-sections were calculated using MOBCAL with the trajectory method parametrised for $\mathrm{N}_{2}$ buffer gas.[36, 37] Input charge distributions were computed at the $\omega$ B97X-D/aug-cc-pVDZ level of theory with the Merz-Singh-Kollman scheme constrained to reproduce the electric dipole moment.[38] Typically, 20 to 30 trajectories were computed, sufficient to give standard deviations of $\pm 1 \AA^{2}$ for the calculated values. Note that the present version of MOBCAL was parametrized for cations and its performance for anions has not been benchmarked.

\section{Results and discussion}

\section{1 $\mathrm{LH}^{-}$}

Arrival time distributions (ATDs) for $\mathrm{LH}^{-}$in $\mathrm{N}_{2}$ buffer gas and $\mathrm{N}_{2}$ buffer gas seeded with $\approx 1 \%$ propan-2-ol are shown in Fig. 2a. Anion-solvent clusters $\left(\mathrm{LH}^{-} \cdot \mathrm{CH}_{3} \mathrm{OH}\right)$ formed in the electrospray source are marked with an asterisk; these cluster anions survive the ion mobility drift region but dissociate to produce $\mathrm{LH}^{-}$in the second ion funnel (ascertained by scanning the quadrupole mass filter and by changing the RF drive voltage applied to second ion funnel to promote cluster dissociation). Both ATDs show evidence for two isomers with distinct collision cross-sections.

$\mathrm{LH}_{2}$ has two obvious deprotonation sites, potentially leading to phenolate and a carboxylate deprotomers (Fig. 3). The latter can have keto and enol tautomers. Furthermore, since each form has $E$ and $Z$ isomers about the central bond joining the two ring systems, there is a total of six possible $\mathrm{LH}^{-}$isomers. The major peak in the ATD for $\mathrm{LH}^{-}$was assigned to the $E$-phenolate isomer since it is the most stable form (Table 1), has the smallest calculated collision crosssection $\left(\Omega_{c}\right.$ in Table 1$)$, and, as detailed below, its action spectrum is consistent with calculated vertical excitation wavelengths for this isomer. The later peak in the ATD for $\mathrm{LH}^{-}$was assigned to the $E$-carboxylate-enol 
Table 1 Calculated relative energies (E), collision cross-sections $\left(\Omega_{c}\right)$, vertical detachment energies (VDE), $\mathrm{S}_{1} \leftarrow \mathrm{S}_{0}$ vertical excitation wavelengths (VE) and oscillator strength, $f$.

\begin{tabular}{cccccc}
\hline Species & $\mathrm{E}\left(\mathrm{kJ} \mathrm{mol}^{-1}\right)^{a, b}$ & $\Omega_{c}\left(\AA^{2}\right)$ & $\mathrm{VDE}(\mathrm{eV})$ & $\mathrm{VE}(\mathrm{nm})^{c}$ & $f^{d}$ \\
\hline LH & & & & & \\
$E$-phenolate & 0 & 168 & 2.87 & $509 / 508$ & 1.0 \\
$Z$-phenolate & 19 & 168 & 2.86 & $508 / 507$ & 1.0 \\
$E$-carboxylate-enol & 8 & 175 & 4.59 & $-/ 488$ & 1.0 \\
$Z$-carboxylate-enol & 30 & 175 & 4.63 & $-/ 488$ & 1.0 \\
$E$-carboxylate-keto & 13 & 169 & 4.04 & $-/ 533$ & 0.8 \\
$Z$-carboxylate-keto & 16 & 169 & 3.99 & $-/ 535$ & 0.8 \\
$\quad$ OxyLH & & & & & \\
$E$-phenolate-keto & 0 & 157 & 3.16 & $516 / 567$ & 1.3 \\
$Z$-phenolate-keto & 25 & 157 & 3.15 & $518 / 574$ & 1.3 \\
$E$-phenolate-enol & 62 & 160 & 2.59 & $-/ 528$ & 1.1 \\
$Z$-phenolate-enol & 83 & 160 & 2.61 & $-/ 529$ & 1.1 \\
$E$-enolate & 71 & 163 & 2.41 & $-/ 623^{e}$ & 0.4 \\
$Z$-enolate & 93 & 163 & 2.44 & $-/ 623$ & 0.4 \\
DMOxyL & & & & & \\
$E$-phenolate-keto & 0 & 165 & 3.12 & $522 / 571$ & 1.4 \\
$Z$-phenolate-keto & 22 & 165 & 3.13 & $523 / 576$ & 1.4 \\
\hline
\end{tabular}

${ }^{a}$ Relative to the lowest energy isomer. ${ }^{b}$ DLPNO-CCSD(T)/aug-cc-pVTZ level of theory. ${ }^{c}$ The first and second values are at the df-CC2/aug-cc-pVTZ and STEOM-DLPNO-CCSD/aug-cc-pVDZ levels of theory, respectively. ${ }^{d}$ Oscillator strengths are from CIS wavefunctions. VDE values are estimated to be within $\pm 0.2 \mathrm{eV}$ of experiment. VDE values were taken from DLPNO-IP-EOM-CCSD calculations as part of the STEOM-DLPNO-CCSD calculations. The experimental VDE for LH ${ }^{-}$ from Ref. 20 is $\approx 3.2 \mathrm{eV}$, which is $\approx 0.3 \mathrm{eV}$ higher than the calculated value for $E$-phenolate- $\mathrm{LH}^{-}$. ${ }^{e}$ The second excited state has $\mathrm{VE}=441 \mathrm{~nm}$ and $f=1 \times 10^{-3}$.

isomer since it is the second lowest energy isomer, is predicted to have a collision cross-section substantially larger than the $E$-phenolate isomer and has an action spectrum consistent with calculated vertical excitation wavelengths for this isomer (see below). It is worth noting that on the basis of the calculated collision cross-sections (Table 1 ), the $Z$ isomers are probably not resolved from their respective $E$ isomers in $\mathrm{N}_{2}$ buffer gas. Furthermore, calculated ground electronic state $E-Z$ isomerization barriers (transition states) for the $E$-phenolate and $E$-carboxylate-enol isomers are 29 and $44 \mathrm{~kJ} \mathrm{~mol}^{-1}$ at the DLPNO-CCSD(T)/aug-ccpVDZ level of theory. Because these barriers are similar to the average thermal energy of the ions at $300 \mathrm{~K}$ (40 $\mathrm{kJ} \mathrm{mol}^{-1}$ assuming a harmonic oscillator vibrational

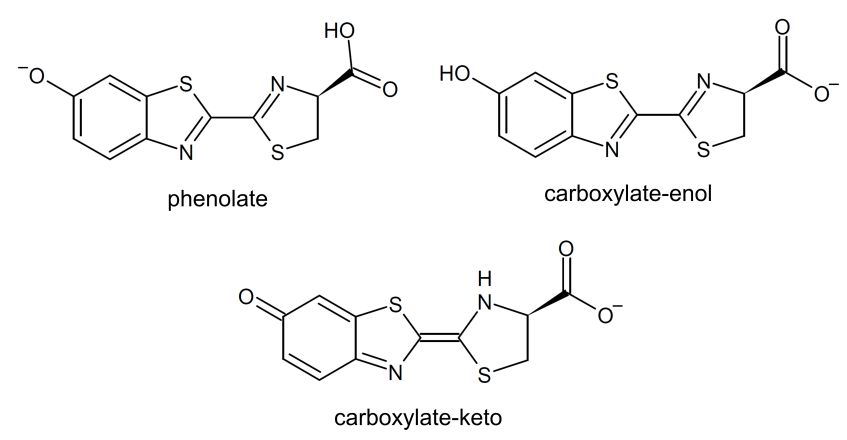

Fig. 3 Deprotomeric and tauotmeric forms of $\mathrm{LH}^{-}$. Molecules are shown as their $E$ isomers with respect to the central bond connecting the two rings. partition function), we expect that the $E$ - $Z$ isomers will rapidly interconvert during their passage through the drift region. [25, 39]

The photodepletion and photodetachment spectra associated with ATD peak 1 ( $E$-phenolate) are shown in Fig. 2b. Both spectra have a maximum at $\approx 505 \mathrm{~nm}$, with the photodepletion spectrum showing an additional maximum at $\approx 530 \mathrm{~nm}$, presumably arising from photodissociation. There was no evidence for photoisomerization in the photoaction ATDs (see Electronic Supplementary Material). These action spectra are consistent with DLPNO-STEOM-CCSD/aug-cc-pVDZ calculations on the $E$-phenolate isomer, which predict an absorption maximum for the $\mathrm{S}_{1} \leftarrow \mathrm{S}_{0}$ transition at $508 \mathrm{~nm}$. The photodetachment band extends well below the calculated vertical detachment energy (VDE) for the assigned isomer at $2.87 \mathrm{eV}(432 \mathrm{~nm})$ presumably due to multiphoton (sequential) absorption - see further discussion in Section 5.

The photodepletion spectrum associated with ATD peak 2 (E-carboxylate-enol) is shown in Fig. 2c. The spectrum has maximum response at $\approx 460 \mathrm{~nm}$ and overlaps with a substantial portion of the action spectrum assigned to the $E$-phenolate isomer (Fig. 2b). The spectrum is consistent with the calculated vertical excitation wavelength for the $E$-carboxylate-enol isomer at $488 \mathrm{~nm}$, but not with the alternative $E$-carboxylateketo tautomer whose vertical excitation wavelength is calculated at $533 \mathrm{~nm}$. Nevertheless, the calculated collision cross-section for the $E$-carboxylate-keto tautomer 
in $\mathrm{N}_{2}$ buffer gas is within $1 \AA^{2}$ of the value for the $E$-phenolate isomer so that both species potentially contribute to ATD peak 1. Furthermore, the calculated vertical excitation wavelength for the $E$-carboxylateketo tautomer $(533 \mathrm{~nm})$ corresponds closely with the additional photodepletion peak in Fig. 2b (see Table 1). The VDE for this species is much larger than for the $E$-phenolate isomer, suggesting that photodissociation would be favoured over electron detachment. We therefore cannot rule out that the $E$-carboxylate-keto tautomer accounts for some fraction of ATD peak 1.
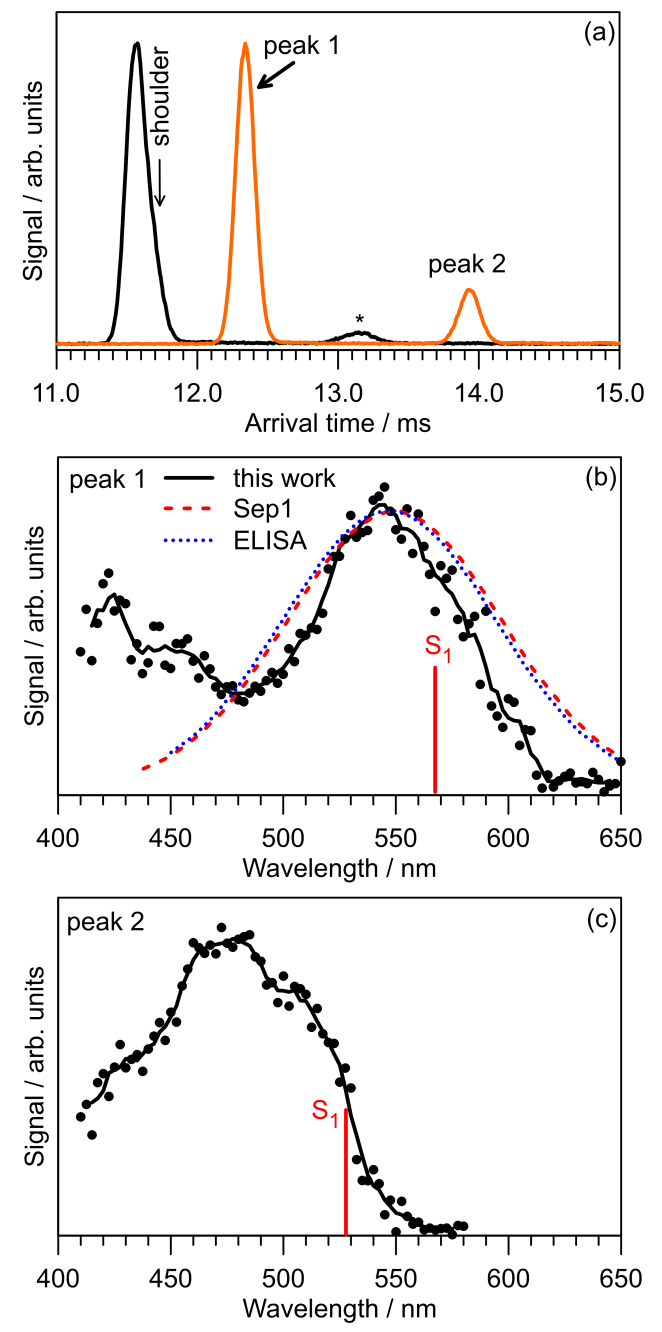

Fig. 4 (a) Arrival time distributions for $\mathrm{OxyLH}^{-}$using $\mathrm{N}_{2}$ (black) and $\mathrm{N}_{2}+\approx 1 \%$ propan-2-ol (orange) buffer gas. The feature denoted by $*$ is from $\mathrm{OxyLH}^{-} \cdot \mathrm{CH}_{3} \mathrm{OH}$ clusters that dissociate in the ion funnel at the end of the drift region. (b) Photodepletion action spectrum for ATD peak 1 (assigned to the $E$-phenolate-keto isomer) and photodissociation spectra (smoothed) from Ref. 19. (c) Photodepletion action spectrum for ATD peak 2 (assigned to the $E$-phenolate-enol isomer). Red bars in (b) and (c) indicate STEOM-DLPNOCCSD/aug-cc-pVDZ vertical excitation wavelengths.
Photodissociation action spectra (smoothed) previously recorded using the Sep1 and ELISA instruments at Aarhus University are shown in Fig. 2d.[18] The Sep1 spectrum agrees closely with the present photodetachment spectrum assigned to the $E$-phenolate isomer (ATD peak 1, Fig. 2b). In contrast, the maximum in the ELISA photodissociation spectrum is shifted by $\approx 40 \mathrm{~nm}$ to longer wavelength. As outlined in the introduction, the difference between the Sep1 and ELISA spectra is attributable to the ELISA instrument recording a larger fraction of ions generated through slower statistical processes. For the experiments described in this paper, slow decomposition and detachment processes should be substantially suppressed in the drift region environment ( $\approx 6$ Torr) due to rapid collisional deactivation. This issue is discussed further in Section 5 .

\section{$4.2 \mathrm{OxyLH}^{-}$}

ATDs for $\mathrm{OxyLH}^{-}$are shown in Fig. 4a. In $\mathrm{N}_{2}$ buffer gas, there is a dominant ATD peak, which has a slight shoulder on the long arrival time edge and which is slightly broader than expected. This hints that two isomers contribute to the peak. In $\mathrm{N}_{2}$ buffer gas seeded with $\approx 1 \%$ propan-2-ol, two peaks (labelled as peak 1 and peak 2) are observed in the ATD with an intensity ratio of approximately 5:1. ATD peaks 1 and 2 were assigned as the $E$-phenolate-keto and $E$ phenolate-enol tautomers (Fig. 5), respectively, based on their relative energies, calculated collision crosssections and agreement between action spectra and calculated excitation wavelengths (see Table 1 ). The calculated barrier for $E-Z$ isomerization on the ground electronic state for the $E$-phenolate-enol tautomer is $19 \mathrm{~kJ} \mathrm{~mol}^{-1}$ (average vibrational energy of the ions at $\approx 300 \mathrm{~K}$ is $33 \mathrm{~kJ} \mathrm{~mol}^{-1}$ ), suggesting the two geometric isomers will rapidly interconvert in the drift region. On the other hand, for the E-phenolate-keto isomer,

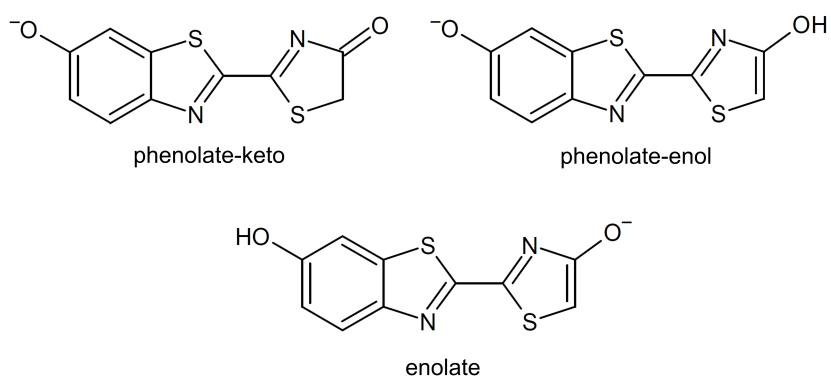

Fig. 5 Deprotomeric and tauotmeric forms of $\mathrm{OxyLH}^{-}$. Molecules are shown in their $E$ isomers with respect to the central bond connecting the two rings. 
the central bond linking the two aromatic units has substantial double bond character and consequently has a higher calculated $E-Z$ isomerization barrier of $62 \mathrm{~kJ} \mathrm{~mol}^{-1}$. However, even if $E$ and $Z$ isomers of this species survive passage through the drift region, they are probably not resolved since they are calculated to have very similar collision cross-sections in $\mathrm{N}_{2}$ (Table 1 ).

Photodepletion action spectra associated with the two ATD peaks in $\mathrm{N}_{2}+\approx 1 \%$ propan-2-ol buffer gas are shown in Fig. $4 \mathrm{~b}$ and c, respectively. The spectra have maxima at $\approx 540 \mathrm{~nm}$ (ATD peak $1, E$-phenolateketo) and $\approx 475 \mathrm{~nm}$ (ATD peak 2, E-phenolate-enol), which are consistent with calculated vertical excitation wavelengths for the two isomers. There was no evidence for photoisomerization in the photoaction ATDs (see Electronic Supplementary Material).

The photodepletion spectrum assigned to the $E$ phenolate-keto isomer (ATD peak 1, Fig. 4b) is similar to the Sep1 and ELISA photodissociation spectra previously recorded at Aarhus University,[19] suggesting that the $E$-phenolate-enol tautomer does not contribute significantly to the earlier Sep1 and ELISA photodissociation action spectra. The onset of a second photodepletion feature in Fig. $4 \mathrm{~b}$ for wavelengths shorter than $\approx 480 \mathrm{~nm}$ is presumably due to direct, single photon photodetachment (the photon energy exceeds the electron detachment threshold) - see further discussion in Section 5.

\section{3 $\mathrm{DMOxyL}^{-}$}

The ATD for DMOxyL ${ }^{-}$, which is shown in Fig. 6a, exhibits one peak irrespective of the buffer gas composition. This was expected as the deprotonated molecule has a single tautomer (phenolate-keto, shown inset in Fig. 6a). The calculated $E-Z$ isomerization barrier for the ground electronic state is $62 \mathrm{~kJ} \mathrm{~mol}^{-1}$ which is larger than the average vibrational energy of the ions at $\approx 300 \mathrm{~K}\left(41 \mathrm{~kJ} \mathrm{~mol}^{-1}\right)$, so that $E$ and $Z$ isomers might survive passage through the drift region. However, both isomers have similar calculated collision cross-sections (Table 1) and are unlikely to be resolved.

The photodepletion action spectrum for $\mathrm{DMOxyL}^{-}$, shown in Fig. 6b, has a maximum at $\approx 550 \mathrm{~nm}$, consistent with the calculated vertical excitation wavelength at $571 \mathrm{~nm}$ (Table 1, STEOM-DLPNO-CCSD/aug-ccpVDZ level of theory). The photodepeletion spectrum resembles the previous Sep1 photodissociation spectrum better than it does the ELISA spectrum. The photodepletion spectrum for $\mathrm{DMOxyL}^{-}$, which certainly has a $E$-phenolate-keto structure, is similar to the photodepletion spectrum assigned to the $E$ -
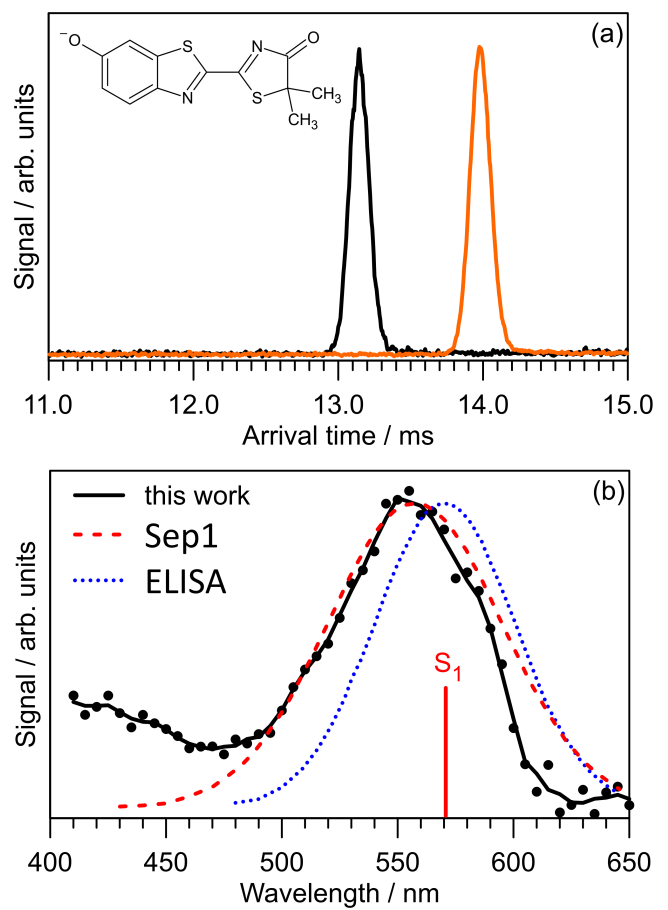

Fig. 6 (a) Arrival time distributions for DMOxyL- using $\mathrm{N}_{2}$ (black) and $\mathrm{N}_{2}+\approx 1 \%$ propan-2-ol (orange) buffer gas. (b) Photodepletion action spectrum associated with the ATD peak and photodissociation spectra (smoothed) from Ref. 19. The red bar in (b) indicates the STEOM-DLPNOCCSD/aug-cc-pVDZ vertical excitation wavelength.

phenolate-keto tautomer for $\mathrm{OxyLH}^{-}$(Fig. 4b), lending support to the ATD peak assignments for $\mathrm{OxyLH}^{-}$.

\section{General discussion}

The ATDs and photodepletion action spectra recorded in this study show that deprotonated D-luciferin and oxyluciferin produced through electrospray ionization can exist in several isomeric forms with distinct action spectra. The occurrence of several isomers is common to a wide range of biochromophores in the gas phase, including $p \mathrm{HBDI}^{-}$(green fluorescent protein chromophore),[40] flavins, [41] para-hydroxycinnamates (photoactive yellow protein chromophore models), [25, 42] and retinoids (photoreceptor in opsin proteins).[43, 44] In the present case, $\mathrm{LH}^{-}$and $\mathrm{OxyLH}^{-}$each exist as at least two isomers that are resolvable using ion mobility, and are assigned as deprotomers and tautomers, respectively. Because the isomers have overlapping action spectra, it is difficult to perform action spectroscopy experiments on a single isomer using techniques without isomer selection. Relative isomer yields from electrospray ionization can depend upon many experimental factors, including the solvent, $\mathrm{pH}$, electrospray geometry and needle voltage, desolvation 
conditions, and collisional treatment of ions as they are introduced into vacuum.[45, 46, 47] Because most gas-phase action spectroscopy instruments are custom designed, it is difficult to know if the isomer distribution for one experiment is the same as for another.

The present action spectra for deprotonated luciferin molecules may deviate from the intrinsic absorption profiles and the earlier Sep1 and ELISA photodissociation action spectra for two reasons: (i) each instrument measures a different fraction of prompt and delayed electron detachment and dissociation, and (ii) the action spectrum for each species extends over the electron detachment threshold, so the number of photons necessary to induce electron detachment varies over the action spectrum. The influence of these factors is discussed below.

The action spectra recorded in the present study have been compared with earlier photodissociation action spectra recorded using the Sep1 and ELISA instruments at Aarhus University. The similarity between the earlier action spectra and those for the most abundant isomer from the present measurements suggest that the electrospray ionization sources at Aarhus University mainly produced the most stable isomer. However, all three experiments are sensitive to different degrees of prompt and delayed photodecomposition. In particular, Sep1 $\left(\approx 10^{-6}\right.$ Torr background pressure) and ELISA ( $\approx 10^{-10}$ Torr background pressure) are high-vacuum experiments and detect charged fragments (Sep1) or neutrals (ELISA) formed over specific time windows after irradiation, with the ELISA detection time window (e.g. $33 \mu$ s to $3 \mathrm{~ms}$ ) considerably longer than the Sep1 window (0 to $3 \mu \mathrm{s})$. On the other hand, the present tandem IMS measurements are performed in a high-pressure environment ( $\approx 6$ Torr), whereby photoactivated ions thermalize over hundreds of nanoseconds (the collision rate in the drift region is $\approx 10^{9} \mathrm{~s}^{-1}$ ). [39, $25]$ Under the drift region conditions, rapid excited state processes occurring on sub-nanosecond timescales, including autodetachment or photoisomerization by passage through a conical intersection, will tend to take place. In contrast, slow statistical dissociation or electron ejection should be suppressed because of collisional deactivation. Generally, the present drift tube action spectra of all three target anions resemble the Sep1 spectra more than the ELISA spectra, presumably because the present experiment is sensitive to rapid photo-induced processes that are also better captured by the Sep1 experiment.

As noted above, the action spectra of the probed luciferin anion species span the expected electron detachment thresholds (see Table 1). Excitation to vibronic levels of the $\mathrm{S}_{1}$ state below the detachment threshold will require absorption of a second photon to cause photodetachment. Therefore, one might expect the photodetachment response will be diminished in the sub-threshold region compared to the supra-threshold region, possibly causing differences between the photodepletion action spectra reported in this study and the intrinsic absorption profiles.

Last, we comment on our failure to observe photofragmentation for any of the luciferin systems. A photodissociation mass spectrometry study on $\mathrm{OxyLH}^{-}$(and $\mathrm{DMOxyL}^{-}$), including with synthetic isotopologues, found the dissociation threshold for the dominant fragment to be $\approx 1.86 \mathrm{eV},[48]$ substantially lower than the VDE and presumably also the adiabatic detachment energy. The major ion fragment at $m / z=175$ (compared with $m / z=249$ for parent $\mathrm{OxyLH}^{-}$) should be detectable in our tandem IMS experiment. Our failure to observe this ion in photoaction ATDs when using the quadrupole mass filter as an ion guide (i.e. no mass filtering) suggests that dissociation is slow and is suppressed in our experiment.

\section{Conclusions}

This work demonstrates that negative-mode electrospray ionization of D-luciferin and oxyluciferin produces several isomeric forms with distinct photodepletion action spectra in the visible region. Electrospray of D-luciferin produces two isomers, assigned to phenolate and carboxylate deprotomers. The deprotomers have overlapping action spectra in the 410$530 \mathrm{~nm}$ range, with the $530-560 \mathrm{~nm}$ range providing a window for photophysics studies targeting the phenolate form using, for example, pump-probe femtosecond spectroscopy. Electrospray of deprotonated oxyluciferin generates two gas-phase isomers assigned as tautomers, with the assignments consistent with measurements on a synthetic derivative of oxyluciferin which has a single tautomeric form. The deprotonated oxyluciferin tautomers have overlapping action spectra in the 410$550 \mathrm{~nm}$ range, with the $550-610 \mathrm{~nm}$ range being a window for photophysical studies of just the keto tautomer. This study complements other tandem IMS investigations of biochromophore ions in the gas phase, which demonstrate that electrospray ionization often generates multiple isomers.

Acknowledgements This research was supported under the Australian Research Council's Discovery Project funding scheme (DP150101427 and DP160100474). Electronic structure calculations were carried out on the High Performance Computing Cluster supported by the Research and Specialist Computing Support service at the University of East Anglia. CK thanks Augustinus Fonden, Niels Bohr Fondet and Oticon 
Fonden for travel support. EC acknowledges support by the Austrian Science Fund (FWF) through a Schrödinger Fellowship (Nr. J4013-N36).

Author contributions Action spectroscopy experiments were performed by CK, JNB and EC in the laboratory of EJB. Calculations were performed by $\mathrm{CK}$ and JNB. The manuscript was drafted by JNB with contributions from all authors.

\section{References}

1. V.R. Viviani, Cell. Mol. Life Sci. 59, 1833 (2002)

2. T. Wilson, J.W. Hastings, Bioluminescence: Living Lights, Lights for Living (Harvard University Press, 2013)

3. G.C. Van de Bittner, E.A. Dubikovskaya, C.R. Bertozzi, C.J. Chang, Proc. Nat. Acad. Sci. 107, 21316 (2010)

4. A. Schena, R. Griss, K. Johnsson, Nat. Comm. 6, 7830 (2015)

5. J. Li, L. Chen, L. Dua, M. Li, Chem. Soc. Rev. 42, 662 (2013)

6. H. Fraga, Photochem. Photobio. Sci. 7, 146 (2008)

7. A. Roda, M. Guardigli, E. Michelini, M. Mirasoli, Trends. Anal. Chem. 28, 307 (2009)

8. D. Scott, E. Dikici, M. Ensor, S. Daunert, Ann. Rev. Anal. Chem. 4, 297 (2011)

9. K. Gomi, N. Kajiyama, J. Biol. Chem. 276, 36508 (2001)

10. H.H. Seliger, W.D. McElroy, Proc. Nat. Acad. Sci. 52, 75 (1964)

11. A.B. Lall, H.H. Seliger, W.H. Biggley, J.E. Lloyd, Science 210, 560 (1980)

12. T. Nakatsu, S. Ichiyama, J. Hiratake, A. Saldanha, N. Kobashi, K. Sakata, H. Kato, Nature 440, 372 (2006)

13. S. Hosseinkhani, Cell. Mol. Life Sci. 68, 1167 (2011)

14. P.H. Liua, P.L. Urban, Anal. Biochem. 593, 54 (2017)

15. V.R. Viviani, F.G.C. Arnoldi, A.J.S. Neto, T.L. Oehlmeyer, E.J.H. Becharae, Y. Ohmiya, Photochem. Photobiol. Sci. 7, 159 (2008)

16. Y. Ando, K. Niwa, N. Yamada, T. Enomoto, T. Irie, H. Kubota, Y. Ohmiya, H. Akiyama, Nat. Photonics 2, $44(2008)$

17. P. Gosset, G. Taupier, O. Crégut, J. Brazard, Y. Mély, K.D. Dorkenoo, J. Léonard, P. Didier, J. Phys. Chem. Lett. 11, 3653 (2020)

18. K. Støchkel, B.F. Milne, S.B. Nielsen, J. Phys. Chem. A 115, 2155 (2011)

19. K. Støchkel, C.N. Hansen, J. Houmøller, L.M. Nielsen, K. Anggara, M. Linares, P. Norman, F. Nogueira, O.V. Maltsev, L. Hintermann, S.B. Nielsen, P. Naumov, B.F. Milne, J. Am. Chem. Soc. 135, 6485 (2013)

20. J.L. Woodhouse, M. Assmann, M.A. Parkes, H. Grounds, S.J. Pacman, J.C. Anderson, G.A. Worth, H.H. Fielding, Phys. Chem. Chem. Phys. 19, 22711 (2017)

21. A.M. Patel, A. Henley, M.A. Parkes, M. Assmann, G.A. Worth, J.C. Anderson, H.H. Fielding, Phys. Chem. Chem. Phys. p. doi:10.1039/D0CP03276J (2020)

22. B.D. Adamson, N.J.A. Coughlan, R.E. Continetti, E.J. Bieske, Phys. Chem. Chem. Phys. 15, 9540 (2013)

23. B.D. Adamson, N.J.A. Coughlan, P.B. Markworth, R.E. Continetti, E.J. Bieske, Rev. Sci. Instr. 85, 123109 (2014)

24. J.N. Bull, E. Carrascosa, N. Mallo, M.S. Scholz, G. da Silva, J.E. Beves, E.J. Bieske, J. Phys. Chem. Lett. 9, 665 (2018)
25. J.N. Bull, G. da Silva, M.S. Scholz, E. Carrascosa, E.J. Bieske, J. Phys. Chem. A 123, 4419 (2019)

26. J.N. Bull, J.T. Buntine, M.S. Scholz, E. Carrascosa, L. Giacomozzi, M.H. Stockett, E.J. Bieske, Faraday Discuss. 217, 34 (2019)

27. G.A. Eiceman, Z. Karpas, H.H. Hill, Ion Mobility Spectrometry, 3rd edn. (CRC Press, 2013)

28. M.J. Frisch, G.W. Trucks, H.B. Schlegel, G.E. Scuseria, M.A. Robb, J.R. Cheeseman, G. Scalmani, V. Barone, B. Mennucci, G.A. Petersson, H. Nakatsuji, M. Caricato, X. Li, H.P. Hratchian, A.F. Izmaylov, J. Bloino, G. Zheng, J.L. Sonnenberg, M. Hada, M. Ehara, K. Toyota, R. Fukuda, J. Hasegawa, M. Ishida, T. Nakajima, Y. Honda, O. Kitao, H. Nakai, T. Vreven, J.A. Montgomery, Jr., J.E. Peralta, F. Ogliaro, M. Bearpark, J.J. Heyd, E. Brothers, K.N. Kudin, V.N. Staroverov, R. Kobayashi, J. Normand, K. Raghavachari, A. Rendell, J.C. Burant, S.S. Iyengar, J. Tomasi, M. Cossi, N. Rega, J.M. Millam, M. Klene, J.E. Knox, J.B. Cross, V. Bakken, C. Adamo, J. Jaramillo, R. Gomperts, R.E. Stratmann, O. Yazyev, A.J. Austin, R. Cammi, C. Pomelli, J.W. Ochterski, R.L. Martin, K. Morokuma, V.G. Zakrzewski, G.A. Voth, P. Salvador, J.J. Dannenberg, S. Dapprich, A.D. Daniels, Ö. Farkas, J.B. Foresman, J.V. Ortiz, J. Cioslowski, D.J. Fox. Gaussian 16 Revision A.03. Gaussian Inc. Wallingford CT 2016

29. F. Neese, WIRES Comp. Mol. Sci. 2, 73 (2012)

30. M. Kállay, Z. Rolik, J. Csontos, P. Nagy, G. Samu, D. Mester, I. Ladjánszki, L. Szegedy, B. Ladóczki, K. Petrov, M. Farkas, B. Hégely. MRCC, A Quantum Chemical Program Suite. www.mrcc.hu

31. J.D. Chai, M. Head-Gordon, Phys. Chem. Chem. Phys. 10, 6615 (2008)

32. T. H. Dunning, Jr., J. Chem. Phys. 90, 1007 (1989)

33. C. Riplinger, B. Sandhoefer, A. Hansen, F. Neese, J. Chem. Phys. 139, 134101 (2013)

34. C. Hättig, F. Weigend, J. Chem. Phys. 113, 5154 (2000)

35. A.K. Dutta, M. Saitow, B. Demoulin, F. Neese, R. Izsák, J. Chem. Phys. 150, 164123 (2019)

36. I. Campuzano, M.F. Bush, C.V. Robinson, C. Beaumont, K. Richardson, H. Kim, H.I. Kim, Anal. Chem. 84(2), 1026 (2012)

37. M.F. Mesleh, J.M. Hunter, A.A. Shvartsburg, G.C. Schatz, M.F. Jarrold, J. Phys. Chem. 100(40), 16082 (1996)

38. B.H. Besler, K.M. Merz, Jr., P.A. Kollman, J. Comp. Chem. 11, 431 (1990)

39. J.N. Bull, M.S. Scholz, E. Carrascosa, G. da Silva, E.J. Bieske, Phys. Rev. Lett. 120, 223002 (2018)

40. E. Carrascosa, J.N. Bull, M.S. Scholz, N.J.A. Coughlan, S. Olsen, U. Wille, E.J. Bieske, J. Phys. Chem. Lett. 9, 2647 (2018)

41. J.N. Bull, E. Carrascosa, L. Giacomozzi, E.J. Bieske, M.H. Stockett, Phys. Chem. Chem. Phys. 20, 19672 (2018)

42. M. Almasian, J. Grzetic, J. van Maurik, J.D. Steill, G. Berden, S. Ingemann, W.J. Buma, J. Oomens, J. Phys. Chem. Lett. 3, 2259 (2012)

43. J.N. Bull, C.W. West, C.S. Anstöter, G. da Silva, E.J. Bieske, J.R.R. Verlet, Phys. Chem. Chem. Phys. 21, $10567(2019)$

44. N.J.A. Coughlan, B.D. Adamson, L. Gamon, K. Catani, E.J. Bieske, Phys. Chem. Chem. Phys. 17, 22623 (2015)

45. H. Xia, A.B. Attygalle, Anal. Chem. 88, 6035 (2016)

46. A.B. Attygalle, H. Xia, J. Pavlov, J. Am. Soc. Mass Spectrom. 28, 1575 (2017) 
47. H. Xia, A.B. Attygalle, J. Am. Soc. Mass Spectrom. 28, $2580(2017)$

48. M.W. Jensen, K. Støchkel, C. Kjær, J.L. Knudsen, O.V. Maltsev, L. Hintermann, P. Naumov, B.F. Milne, S.B. Nielsen, Int. J. Mass Spectrom. 365-366, 3 (2014) 
Noname manuscript No.

(will be inserted by the editor)

Electronic Supplementary Material for

'Action Spectroscopy of Isomer-Selected Luciferin Anions'

Christina Kjær • James N. Bull • Eduardo

Carrascosa · Steen Brøndsted Nielsen • Evan

J. Bieske

C. Kjær \& S. Brøndsted Nielsen

Department of Physics and Astronomy, Aarhus University, Aarhus 8000, Denmark

J. N. Bull

School of Chemistry, Norwich Research Park, University of East Anglia, Norwich NR4 7TJ,

United Kingdom

E. Carrascosa

Laboratoire de Chimie Physique Moléculaire, École Polytechnique Fédérale de Lausanne, EPFL SB ISIC LCPM, Station 6, CH-1015 Lausanne, Switzerland

E. J. Bieske

School of Chemistry, University of Melbourne, Parkville, VIC 3010, Australia 


\section{S1 Photoaction ATDs}

Light-off and photoaction (light-on - light-off) ATDs for $\mathrm{LH}^{-}, \mathrm{OxyLH}^{-}$and $\mathrm{DMOxyL}^{-}$are shown in Fig. S1. In all cases the photoaction signal was less than a few percent and there was no photoisomerization since there were no growth features in the photoaction ATDs. It worth noting that each ATD peak could be cleanly gated during the IMS-photo-IMS measurements, confirming that there is no rapid interconversion between the isomers in the drift region. 

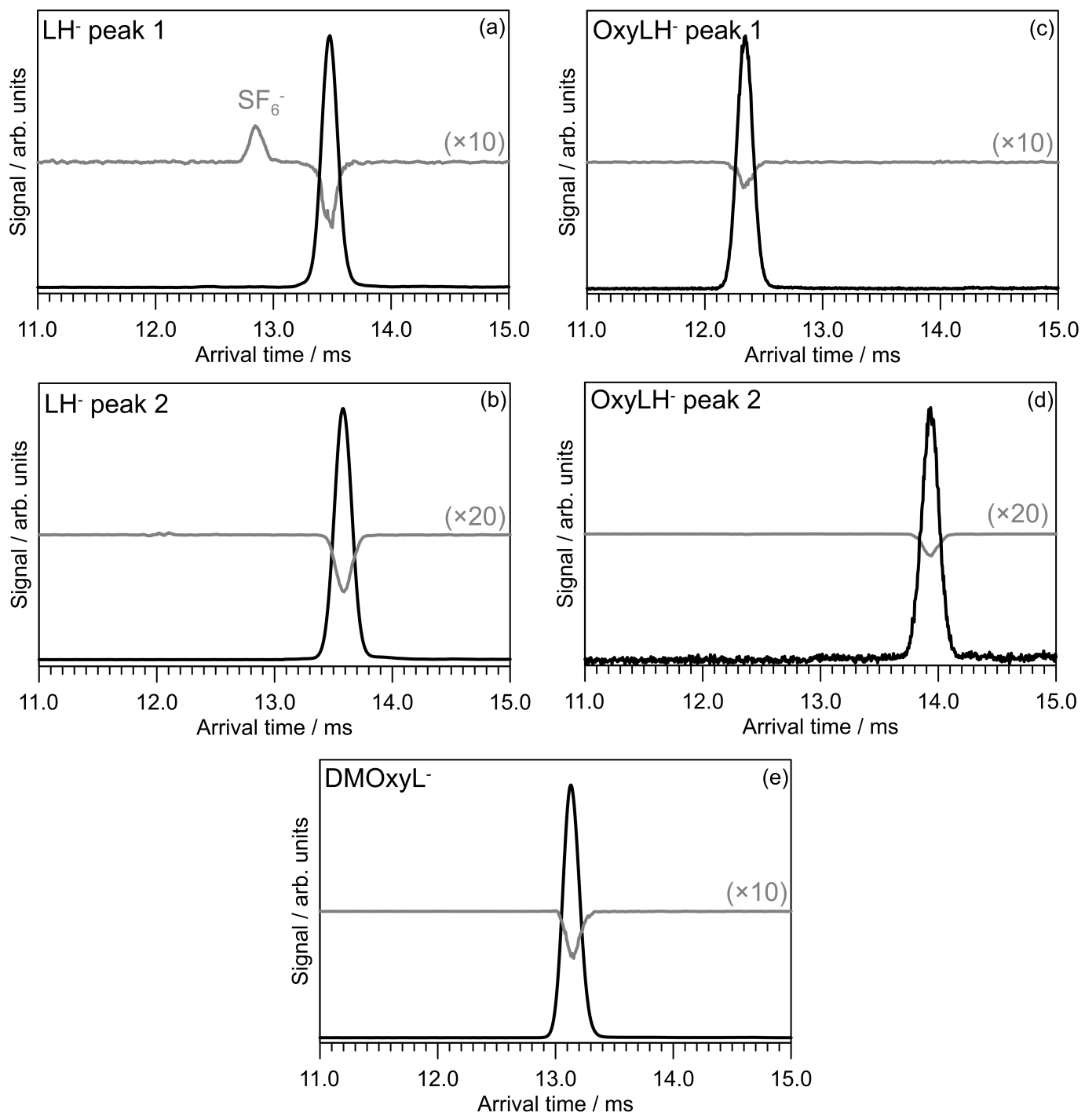

Fig. S1 Light-off (black) and photoaction (grey) ATDs for: (a) $\mathrm{LH}^{-}$ATD peak 1 recorded in $\mathrm{N}_{2}+\mathrm{SF}_{6}$ [quadrupole mass filter set as an ion guide] at $520 \mathrm{~nm}$, (b) $\mathrm{LH}^{-}$ATD peak 2 recorded in pure $\mathrm{N}_{2}$ at $480 \mathrm{~nm}$, (c) OxyLH ${ }^{-}$ATD peak 1 recorded in $\mathrm{N}_{2}+\approx 1 \%$ propan-1-ol at $550 \mathrm{~nm}$, (d) $\mathrm{OxyLH}^{-}$ATD peak 2 recorded in $\mathrm{N}_{2}+\approx 1 \%$ propan-1-ol at $500 \mathrm{~nm}$, and (e) DMOxyLrecorded in pure $\mathrm{N}_{2}$ at $550 \mathrm{~nm}$. Note, ATD peaks in this figure may be shifted compared with ATDs in the paper because of slight changes in buffer gas pressure and composition from day to day. 